\title{
Structure-Dependent Chiroptical Properties of Twisted Multilayered Silver Nanowire Assemblies
}

Wenbing $\mathrm{Wu}^{1}$, Yann Battie ${ }^{2}$, Vincent Lemaire ${ }^{1}$, Gero Decher ${ }^{1,3,4}$, Matthias Pauly ${ }^{1 *}$

1. Université de Strasbourg, CNRS, Institut Charles Sadron UPR22, 67000 Strasbourg, France

2. Université de Lorraine, $L C P-A 2 M C, 57000$ Metz, France

3. International Center for Frontier Research in Chemistry, 67083 Strasbourg, France

4. International Center for Materials Nanoarchitectonics, Tsukuba, Ibaraki 305-0044, Japan

Corresponding author: matthias.pauly@ics-cnrs.unistra.fr 


\section{Materials and Methods}

\section{Materials}

Poly(ethyleneimine) (PEI, $\left.\overline{\mathrm{M}_{\mathrm{n}}} \approx 60,000 \mathrm{~g} / \mathrm{mol}\right)$ ), Poly(sodium 4-styrene sulfonate) (PSS, $\overline{\mathrm{M}_{\mathrm{w}}} \approx 70,000 \mathrm{~g} / \mathrm{mol}$ ), Poly(allylamine hydrochloride) (PAH, $\overline{\mathrm{M}_{\mathrm{w}}} \approx 15,000 \mathrm{~g} / \mathrm{mol}$ ) were purchased from Sigma-Aldrich, Sodium Chloride was purchased from Carl Roth, silver nanowires were purchased from ACS Materials $(\mathrm{d} \approx 60 \mathrm{~nm}, \mathrm{~L} \approx 3-5 \mu \mathrm{m})$ and glass slides from Hellma. All the chemicals were used without further purification. Ultrapure water with a resistivity of $18.2 \mathrm{M} \Omega . \mathrm{cm}$ was obtained by purification with a Milli-Q Gradient system (Millipore) and was used directly after production.

Poly(ethylene imine) (PEI) solutions were freshly prepared by direct dissolution of $2.5 \mathrm{mg} / \mathrm{mL}$ of the polymer in ultrapure water. Poly(sodium 4-styrene sulfonate) (PSS) solutions were prepared in $\mathrm{NaCl}$ solution $(0.5 \mathrm{M})$ at concentration of $0.618 \mathrm{mg} / \mathrm{mL}$. Poly(allylamine hydrochloride) (PAH) solutions were prepared in $\mathrm{NaCl}$ solution $(0.5 \mathrm{M})$ at concentration of $0.285 \mathrm{mg} / \mathrm{mL}$. The AgNW suspension was prepared at a concentration of $0.2 \mathrm{mg} / \mathrm{ml}$ by diluting 50 times the commercial solution (originally in ethanol at $10 \mathrm{mg} / \mathrm{ml}$ ) with milli-Q water.

\section{Oriented deposition by Grazing Incidence Spraying}

The glass slides were cleaned with ethanol and Milli-Q water and activated for 3 minutes by plasma. The polyelectrolytes were orthogonally sprayed for 6 seconds using Air-Boy spraying bottles followed by rinsing with pure water for 10 seconds. AgNWs were deposited on the polymer-coated substrates for 200 seconds using a home-made spraying system consisting of a gas flow controller to adjust the air flow rate (model Red-Y, Voegtlin), a liquid handling pump (model $\mathrm{M} 50, \mathrm{VICl}$ ), and 2-fluid nozzles (internal diameter: $300 \mu \mathrm{m}$, Spraying Systems). The liquid flow rate was set to $1 \mathrm{~mL} / \mathrm{min}$ and the air flow to $30 \mathrm{~L} / \mathrm{min}$ for the AgNWs. The angle between the spray cone main axis and the receiver substrate was $15^{\circ}$. The deposition was followed by a rinsing step with water for 100 seconds using an equivalent nozzle fed with air flow at $25 \mathrm{~L} / \mathrm{min}$ and water flow rate at $10 \mathrm{~mL} / \mathrm{min}$, before drying using air flow.

\section{Variation of the interlayer spacing}

The spacing between the AgNW layers was varied by changing the number of PSS/PAH layer pairs in the deposition sequence $\mathrm{PEI} / \mathrm{PSS} / \mathrm{PAH} / \mathrm{PSS} / \mathrm{PEI} / \mathrm{AgNW}_{\mathrm{S}} / \mathrm{PEI} /(\mathrm{PSS} / \mathrm{PAH})_{n} / \mathrm{PSS} / \mathrm{PEI} / \mathrm{AgNW}_{\mathrm{S}}$. The spacing $\mathrm{d}$ between the 
AgNW layers varies proportionally to the number of PSS/PAH layer pairs $n$ (Figure 3b). Many series of samples have been used to study the effect of the interlayer spacing on the optical properties, from which two series are used in this paper: Series 1 has been prepared for the cross-section imaging presented in Figure $2 \mathrm{a}$ and Figure $2 \mathrm{~b}$ with a round number of PSS/PAH layer pairs, while the number of PSS/PAH layer pairs has been adjusted to reach a round number for the spacing for Series 2 used for the optical characterization. The average and standard deviation of the spacing are given for Series 1, as calculated from the interlayer spacing averaged from 10-20 different positions on the cross-section SEM images.

Table S1. Interlayer spacing for the different number of PSS/PAH layer pair used in the two series of samples on which this study is based.

\begin{tabular}{cc|cc}
\multicolumn{2}{c|}{ Series 1 } & \multicolumn{2}{c}{ Series 2 } \\
$\mathbf{n}$ & $\mathbf{d}(\mathbf{n m})$ & $\mathbf{n}$ & $\mathbf{d}(\mathbf{n m})$ \\
\hline 5 & $13.2 \pm 3.1$ & 5 & 13 \\
15 & $29.6 \pm 5.1$ & 15 & 30 \\
30 & $64.0 \pm 6.7$ & 28 & 60 \\
60 & $124.8 \pm 10.4$ & 48 & 100 \\
100 & $213.8 \pm 15.8$ & 72 & 150 \\
& & 96 & 200 \\
\hline
\end{tabular}

\section{Characterization}

A FEG-SEM (Hitachi SU8010) operating at $1 \mathrm{keV}$ using the SE-in lens detector was used for electron microscopy. Cross-section of the samples have been prepared with a Hitachi IM4000Plus ion milling system with a low-energy $\mathrm{Ar}^{+}$ion beam (6 keV).

CD spectra were measured with a commercial CD spectrometer (model J-1700) from Jasco (Japan). Extinction was measured with a Cary 5000 spectrometer from Agilent (USA) equipped with Glan-Taylor polarizers.

Two polarimeters are used to measure the Mueller matrix of samples: a homemade dualrotating compensator ellipsometer (R2C) and a phase modulated ellipsometer (UVISEL, Horiba). The depolarization parameter of the samples is measured in transmission by the R2C ellipsometer. This measurement reveals that the depolarization parameter is smaller than 0.007 for all samples. In other words, the depolarization can be neglected. The R2C has a smaller sensitivity and signal to noise ratio than the UVISEL ellipsometer. Thus, we deliberately measure the Mueller matrix spectra in transmission with the UVISEL ellipsometer. The UVISEL 
ellipsometer only records the first three columns of the Mueller matrix. The last column is obtained by using the procedure proposed by Arteaga et al. for a non-depolarizing sample. ${ }^{1}$

\section{Modeling of the optical properties}

The Mueller matrix of the nanostructures is simulated in transmission according to the Berreman transfer matrix formalism (BTM). ${ }^{2}$ The Berreman transfer matrix is deduced from the following equation:

$$
\frac{\partial \psi}{\partial z}=i k_{0} \Delta \psi
$$

where $\psi=\left(\begin{array}{c}E_{x} \\ E_{y} \\ H_{x} \\ H_{y}\end{array}\right)$ and $k_{0}=\frac{\omega}{c} . E_{x, y}$ and $H_{x, y}$ are the components of the electric and magnetic fields of light, respectively. $\Delta$ is a $4 \times 4$ matrix which does not depend on $z$ as long as the medium is homogeneous. By considering a non-magnetic and non-gyrotropic layer, the matrix $\Delta$ is given for a normal incidence (along $\mathrm{z}$ ) by:

$$
\Delta=\left(\begin{array}{cccc}
0 & 0 & 0 & 1 \\
0 & 0 & -1 & 0 \\
\frac{\varepsilon_{23} \varepsilon_{31}}{\varepsilon_{33}}-\varepsilon_{21} & \frac{\varepsilon_{23} \varepsilon_{32}}{\varepsilon_{33}}-\varepsilon_{22} & 0 & 0 \\
\varepsilon_{11}-\frac{\varepsilon_{13} \varepsilon_{31}}{\varepsilon_{33}} & \varepsilon_{12}-\frac{\varepsilon_{13} \varepsilon_{32}}{\varepsilon_{33}} & 0 & 0
\end{array}\right)
$$

$\varepsilon_{i, j}$ are the element of the dielectric tensor of the anisotropic medium.

By solving equation (1), we obtain:

$$
\psi\left(z+d_{n}\right)=\exp \left(i k_{0} \Delta \mathrm{d}_{n}\right) \psi(z)=T_{P, n}\left(\mathrm{~d}_{n}\right) \psi(z)
$$

The matrix $T_{P}(d)=\exp \left(i k_{0} \Delta \mathrm{d}\right)$ connects the in-plane components of electric and magnetic fields between two interfaces of anisotropic layers separated by a distance $d$.

By considering that the structure consists in a stack of $\mathrm{N}$ layer of thickness $\mathrm{d}_{\mathrm{N}}$, we can introduce a matrix $T$ that connects the $p$ and s component of the incident fields to the transmitted fields:

$$
\left(\begin{array}{c}
E_{i, s} \\
E_{r, s} \\
E_{i, p} \\
E_{r, p}
\end{array}\right)=T\left(\begin{array}{c}
E_{t, s} \\
0 \\
E_{t, p} \\
0
\end{array}\right)
$$

where

$$
T=L_{a}^{-1} \prod_{n=1}^{N} T_{P, n}\left(-\mathrm{d}_{n}\right) \mathrm{L}_{f}
$$

The index $\mathrm{i}, \mathrm{r}$ and $\mathrm{t}$ are related to the incident, reflected and transmitted electric fields, respectively. By considering air as the ambient medium, the matrix $L_{a}^{-1}$ that connects the 
incident $\psi$ vector to the $\mathrm{s}$ and $\mathrm{p}$ components of the incident and reflected electric fields is given by:

$$
L_{a}^{-1}=0.5\left(\begin{array}{cccc}
0 & 1 & -1 & 0 \\
0 & 1 & 1 & 0 \\
1 & 0 & 0 & 1 \\
-1 & 0 & 0 & 1
\end{array}\right),
$$

The exit matrix $\mathrm{L}_{f}$ which transforms the transmitted $\psi$ vector into the $\mathrm{s}$ and $\mathrm{p}$ components of the transmitted electric field is given by:

$$
\mathrm{L}_{f}=\left(\begin{array}{cccc}
0 & 0 & 1 & 0 \\
1 & 0 & 0 & 0 \\
-1 & 0 & 0 & 0 \\
0 & 0 & 1 & 0
\end{array}\right)
$$

Equation (5) allows calculating the Fresnel coefficients, the Jones matrix and then the Mueller matrix of multilayer structures.

In the following, we consider thin films composed of oriented AgNW layers oriented at a varying angle $\alpha$ (fixed interlayer spacing $d=13 \mathrm{~nm}$ ) as well as thin films composed of layers oriented with a varying interlayer spacing $d$ (fixed angle $\alpha=45^{\circ}$ ). The underlying substrate is a 1-mm thick transparent silica substrate. These structures, depicted in Figure S1, are composed of two building blocks, namely a layer of oriented AgNWs of constant thickness and varying orientation and an isotropic polymeric layer of varying thickness.
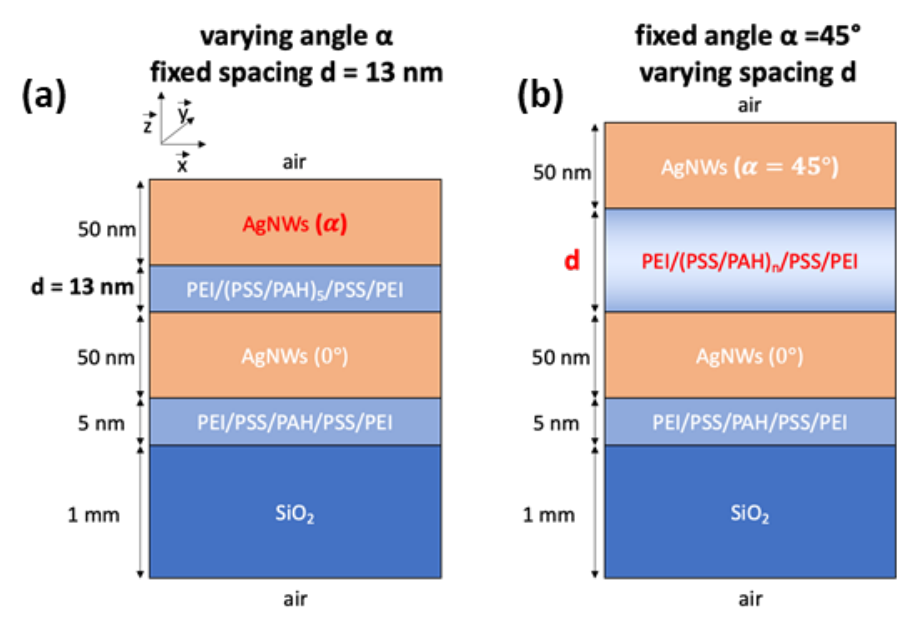

Figure S1. Structures of the 2-layer AgNW thin films used for the modeling. The optical axis of AgNW layer is in the $(\vec{x}, \vec{y})$ plane, the wires being oriented along the $\vec{x}$ axis in the bottom layer and at an angle $\alpha$ in the top layer, with a variable spacing $d$ between the AgNW layers.

According to equation (2), the BTM formalism is based on the dielectric function of each layer, which are deduced from ellipsometric measurements in reflection performed on films 
deposited on silicon substrate. The dielectric constant of the organic layers is set to 2.34. The oriented AgNW monolayer has a uniaxial anisotropy with an optical axis parallel to the AgNW long axis. The ordinary $\left(\varepsilon_{o}\right)$ and extraordinary $\left(\varepsilon_{e}\right)$ effective dielectric functions of the aligned AgNW layer are shown in Figure S2.
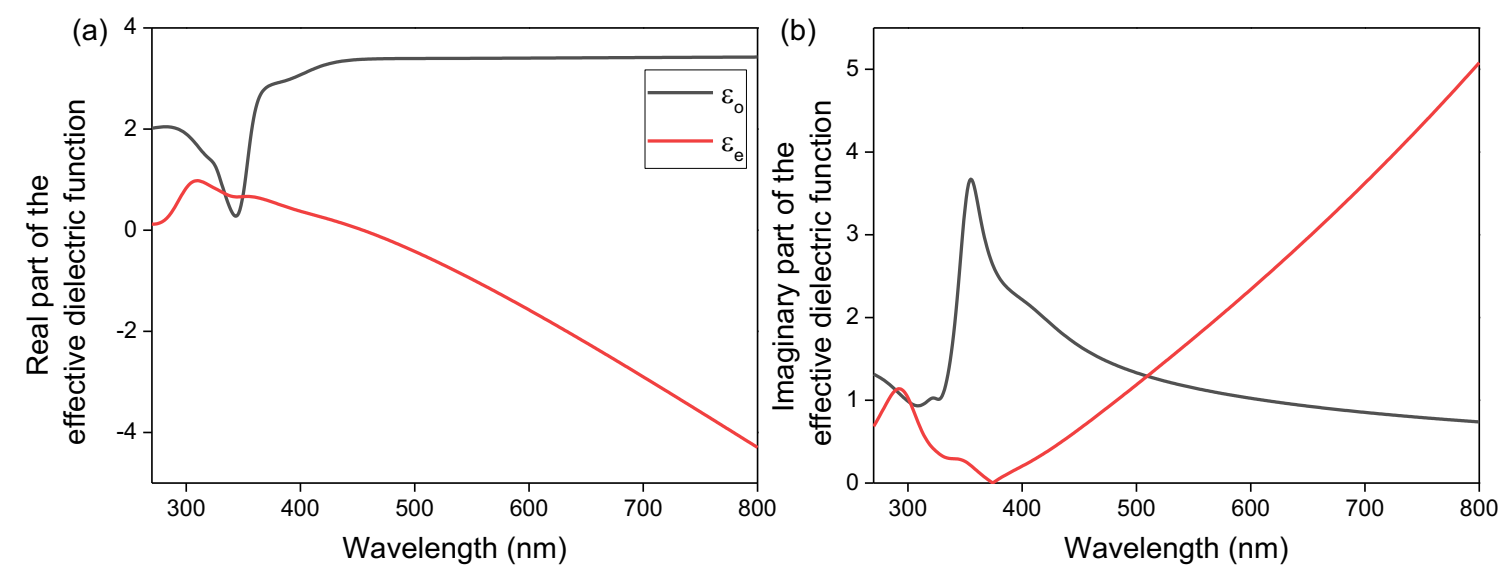

Figure S2. (a) real part and (b) imaginary part of the ordinary and extraordinary effective dielectric function of AgNW layer.

The ordinary effective dielectric function of AgNWs layer is dominated by a plasmon band located at $355 \mathrm{~nm}$. On the contrary, no plasmon band is observed in the extraordinary effective dielectric function. As $\varepsilon_{e} \varepsilon_{o}<0$, the AgNW layer is a hyperbolic metamaterial in the visible/NIR spectral range. The linear or circular dichroism and birefringence are then calculated from the Mueller matrix by using the differential Mueller matrix decomposition. ${ }^{3}$

\section{On the origin of the oscillations in the CD spectra}

To discuss the origin of oscillations observed in the CD spectra (Figure 3 ) for the structure shown in Figure S1(b) with large spacing d between the oriented AgNW layers, we perform 3 different simulations:

1. the Mueller matrix of an isotropic polymer layer on a silica substrate is simulated in transmission at normal incidence. The Mueller matrix of this structure is the identity matrix and the $C D$ is equal to 0 .

2. the CD spectrum of the structure shown in Figure S1(b) (2 AgNW layers oriented at $45^{\circ}$ with a polymer layer of variable spacing $d$ between these AgNW layers) is calculated without taking into account back-reflections at each interface. This spectrum is reported on Figure S3. 
3. the CD spectrum of the same structure is calculated by vanishing the coherence of light inside the polymer layer. This spectrum is also reported on Figure S3.

The simulations 2 . and 3. are performed according to the procedure described by Nichols et $a l .4,5$ These spectra are compared to the those obtained with back-reflection at interfaces and with a coherent transmission of light in the polymer layer for 2 different spacings $d$ (as in Figure 3).

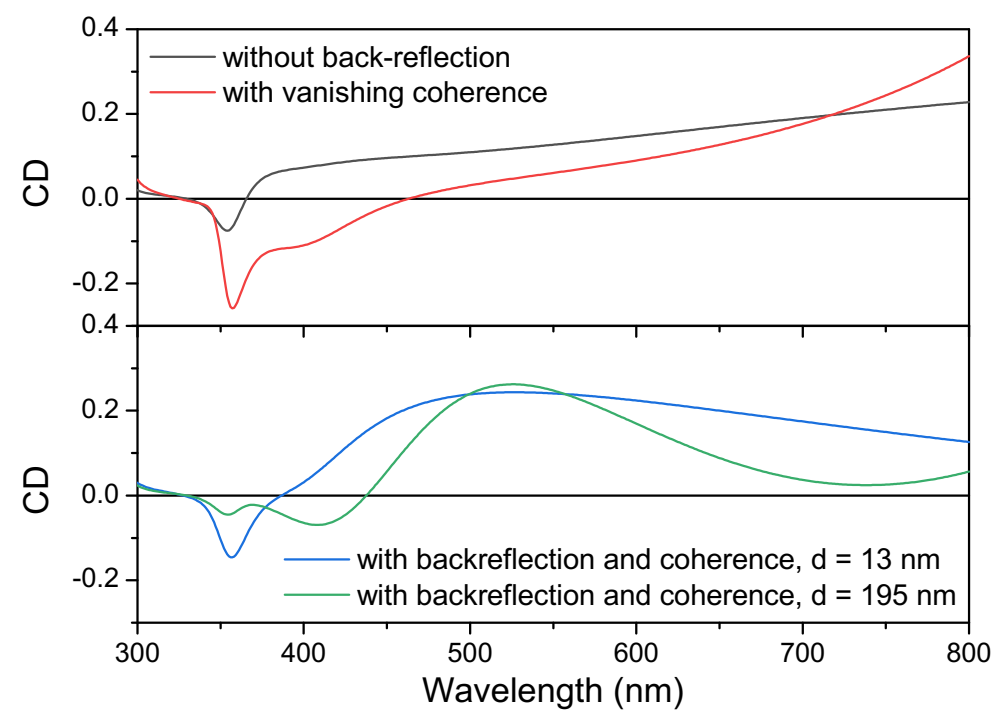

Figure S3. (top) CD spectra of the structure shown in Figure S1(b) simulated without considering backreflection at interfaces and by vanishing the coherence inside the polymer layer (bottom) CD spectra with back-reflection at interfaces and with a coherent transmission of light in the polymer layer for 2 different spacings $d$ between the AgNW layers oriented at $45^{\circ}$.

The simulated spectra without back-reflection at interfaces and with a vanishing coherence inside the polymer layer (Figure S3) do not depend on the thickness $d$ of the polymer layer and do not exhibit any interference pattern, whereas oscillations in the CD spectra are clearly visible when light is coherently transmitted in the polymer layer and back-reflections at interfaces are considered. These results confirm that the oscillations observed in the CD spectra of Figure 3 come from the interference inside the polymer layer. This interference pattern can only be observed if the polymer layer is sandwiched between two anisotropic layers. 


\section{Supplementary Figures}

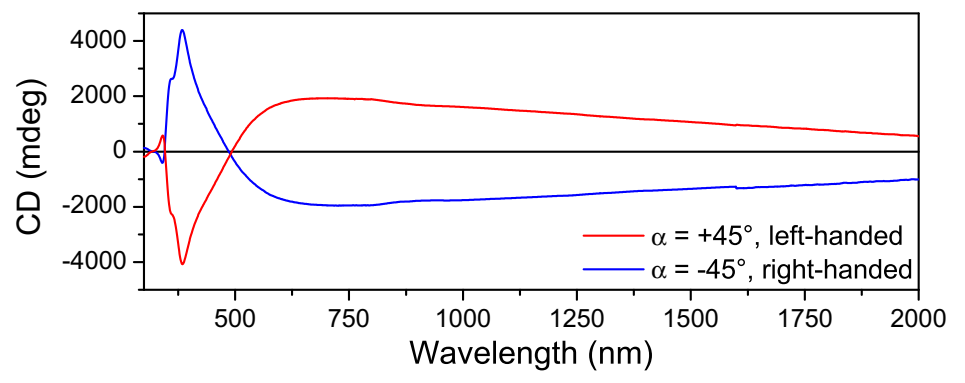

Figure S4. CD spectra of 2-layer samples oriented at an angle $\alpha=+45^{\circ}$ (left-handed) and $\alpha=-45^{\circ}$ (righthanded).

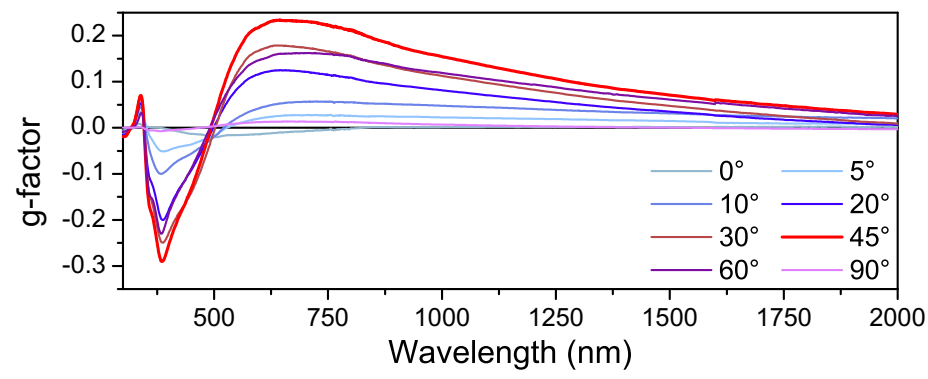

Figure S5. g-factor spectra of 2-layer samples oriented at a varying angle $\alpha$ calculated from the CD and the extinction spectra: $g=\frac{\Delta A}{A}=\frac{\theta(\text { mdeg })}{32982 A}$. 
a

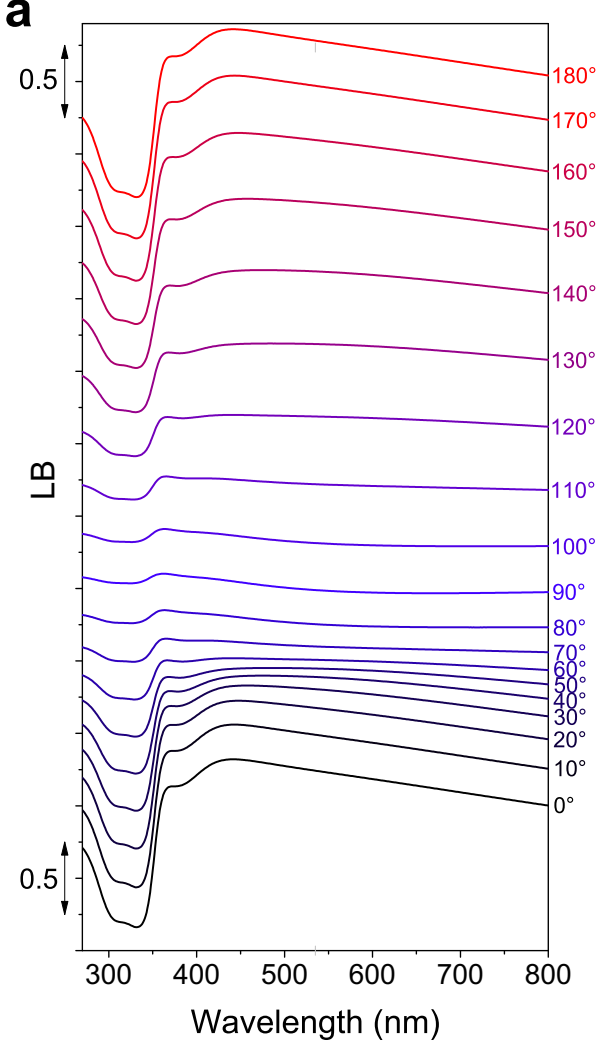

C

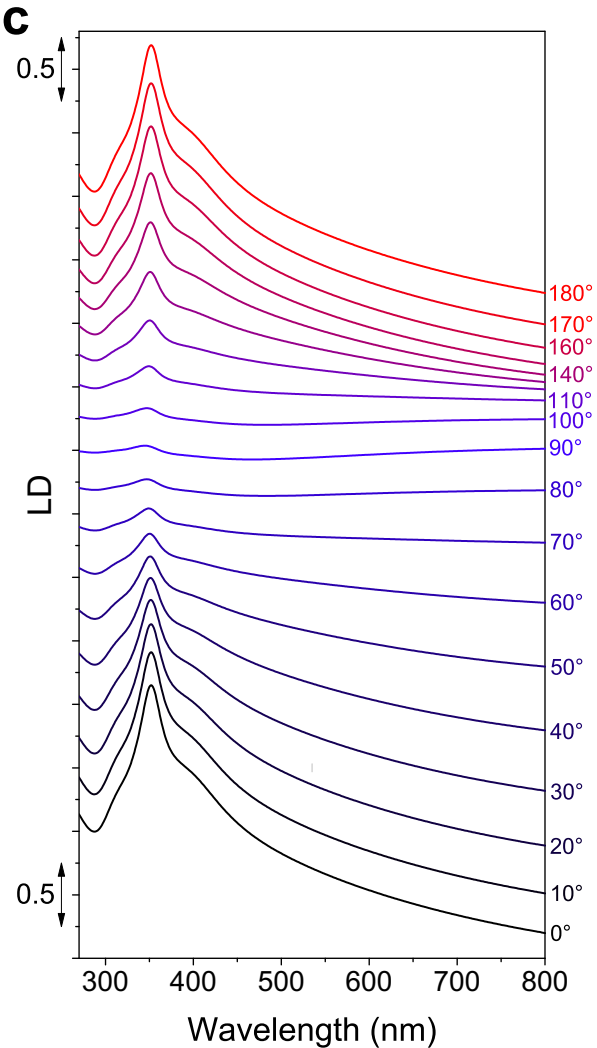

b

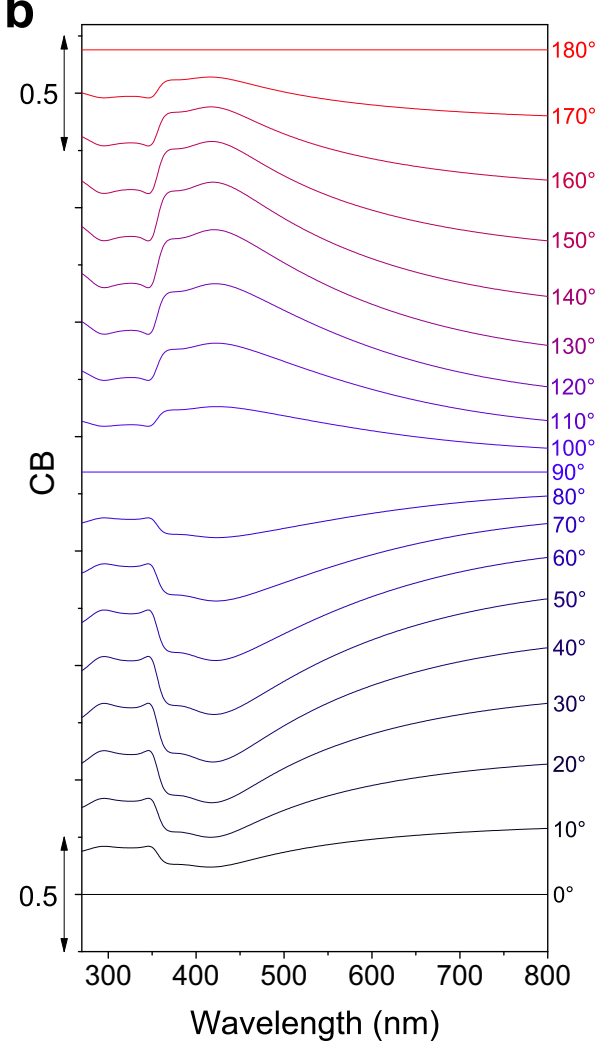

d

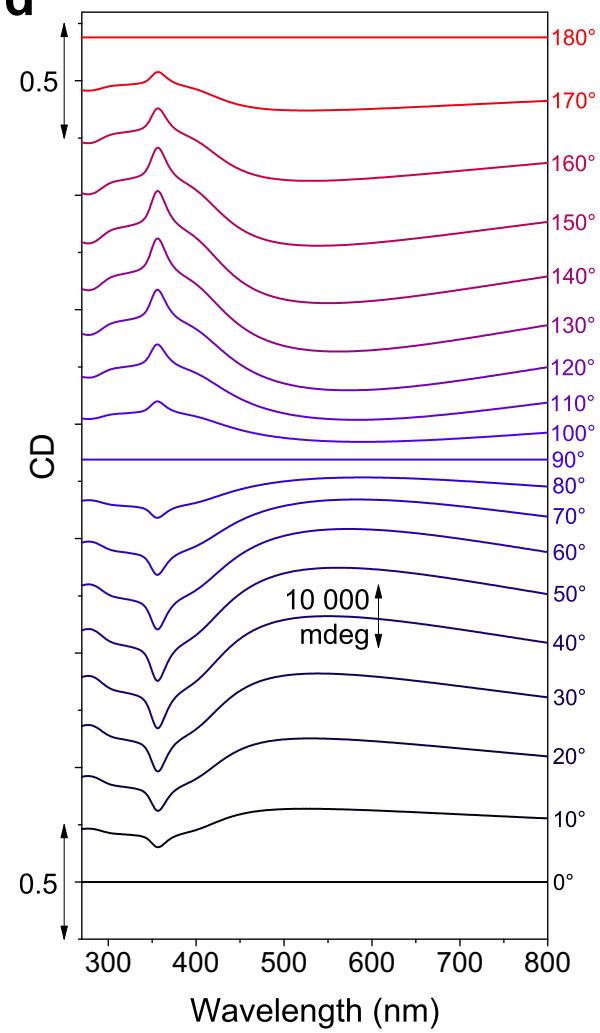

Figure S6. a) Linear birefringence (LB), b) circular birefringence (CB), c) linear dichroism (LD) and d) circular dichroism (CD) spectra modeled using the transfer matrix approach for 2-layer samples oriented at a fixed spacing $(d=13 \mathrm{~nm})$ with a varying angle $\alpha$. The different spectra are stacked with a constant spacing of 0.28 for $L B$ and $L D$ and of 0.21 for $C B$ and $C D$, the $y$-scale bar being the same for $L B$ and $L D$ and for $C B$ and $C D$. 

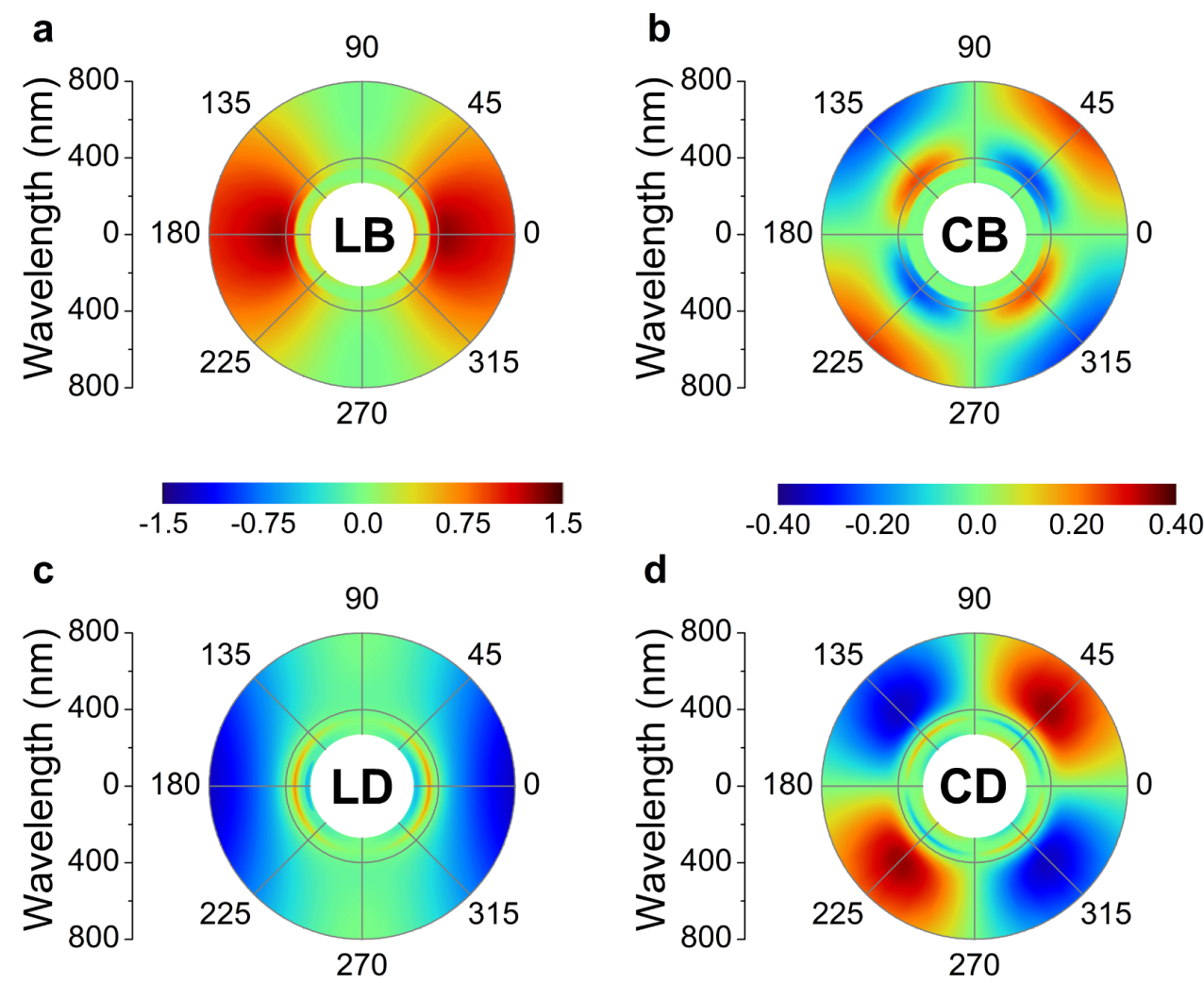

Figure 57. Color polar plot of the a) linear birefringence (LB), b) circular birefringence (CB), c) linear dichroism (LD) and d) circular dichroism (CD) spectra modeled using the transfer matrix approach for 2-layer samples oriented at a fixed spacing $(d=13 \mathrm{~nm})$ with a varying angle $\alpha$. The spectra are identical to those of Fig. S5 plotted as color polar plots. LB and LD share the color scale on the left, while CB and $\mathrm{CD}$ share the color scale on the right. 


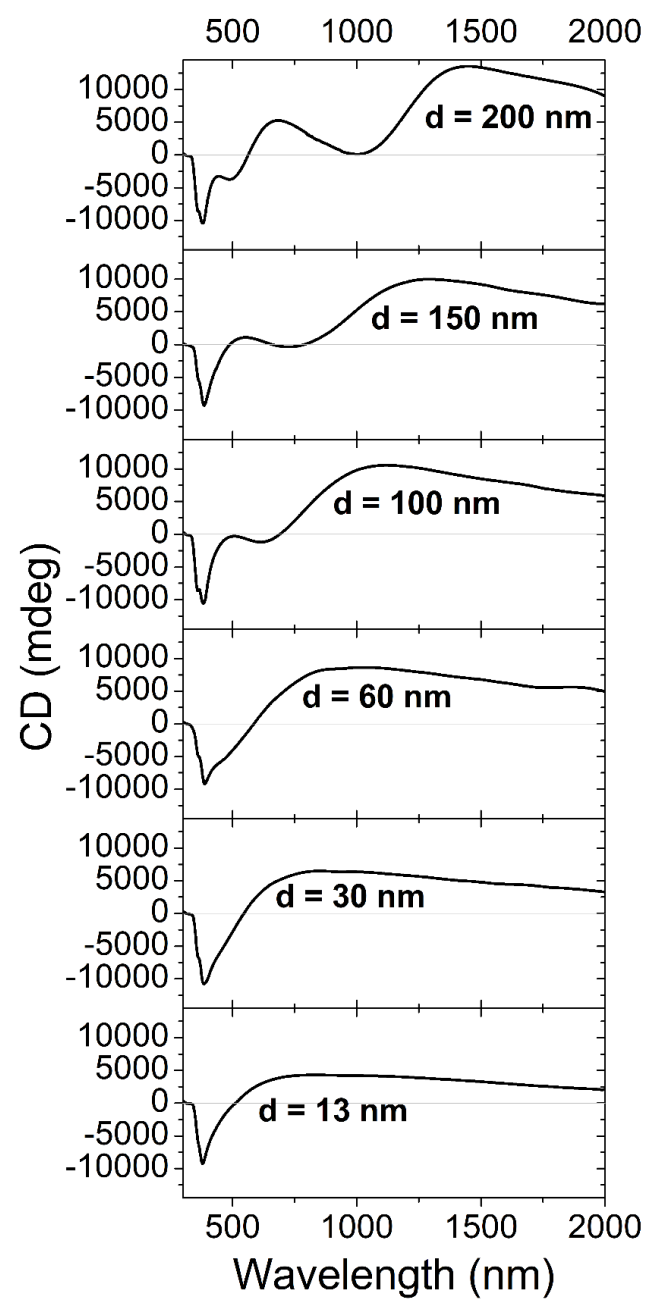

Figure S8. CD spectra measured for 2-layer samples oriented at a fixed angle $\left(\alpha=45^{\circ}\right)$ with a varying interlayer spacing $\mathrm{d}$.

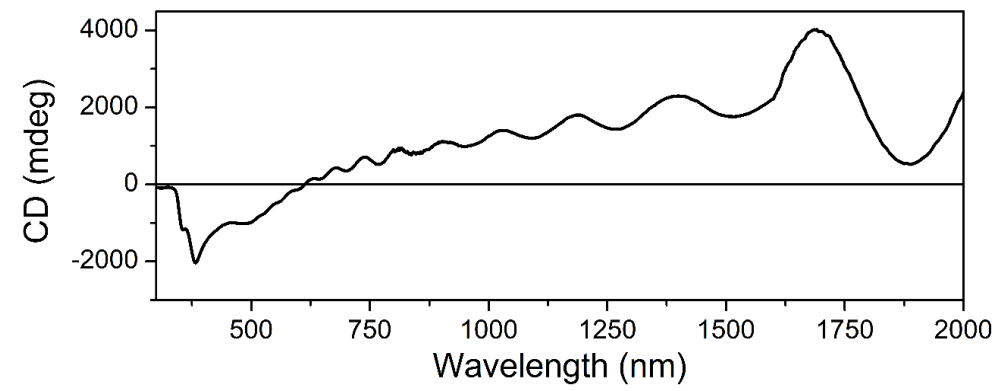

Figure S9. CD spectra of 2-layer samples oriented at an angle $\alpha=+45^{\circ}$ and separated by a $2-\mu \mathrm{m}$ thick PMMA spacer deposited by spin-coating. 

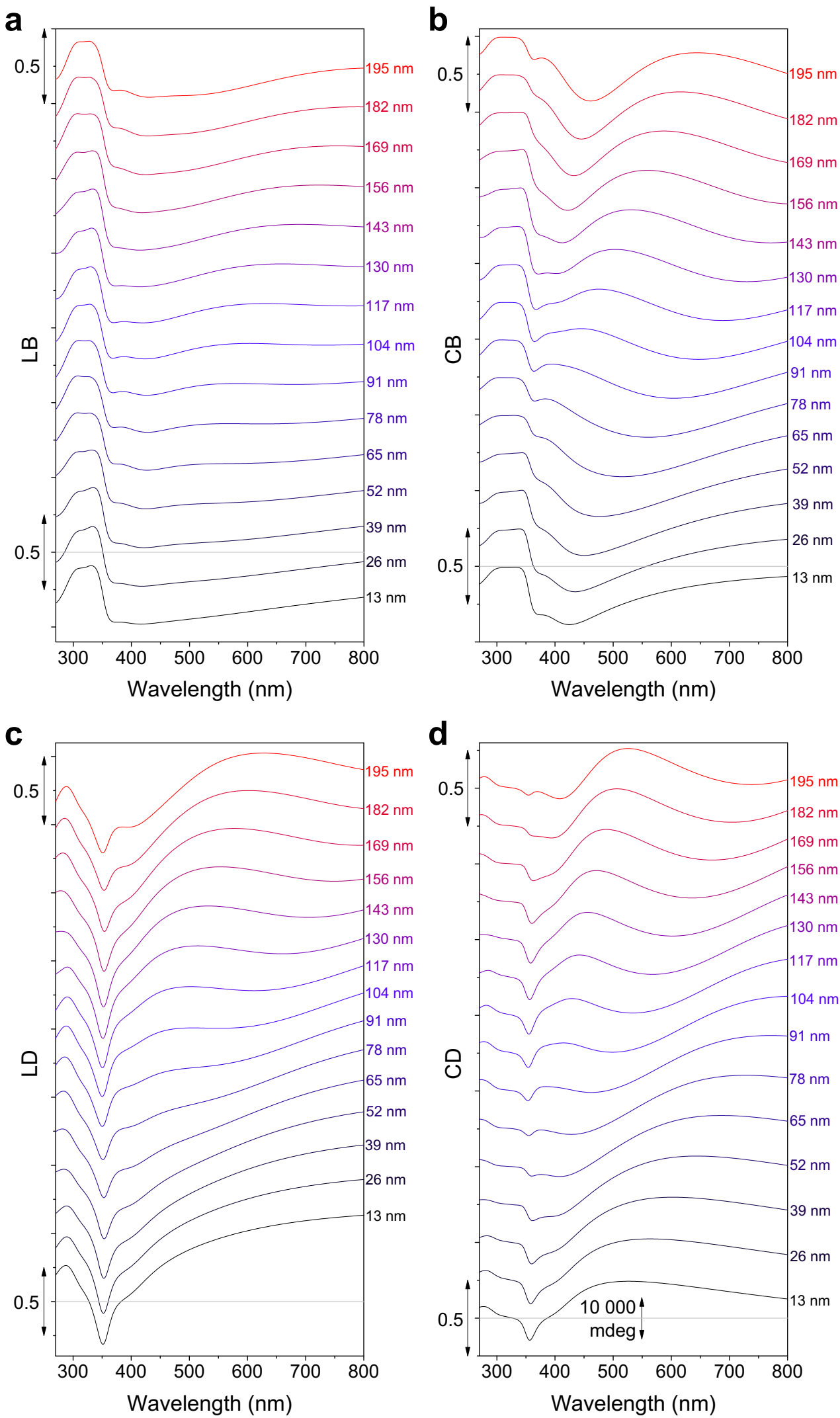

Figure S10. a) Linear birefringence (LB), b) circular birefringence (CB), c) linear dichroism (LD) and d) circular dichroism (CD) spectra modeled using the transfer matrix approach for 2-layer samples oriented at a fixed angle $\left(\alpha=45^{\circ}\right)$ with a varying interlayer spacing $\mathrm{d}$. The different spectra are stacked with a constant spacing of 0.25 , the $y$-scale bar being the same for all spectra. The grey line indicates the $y=0$ axis for the bottom spectrum. 

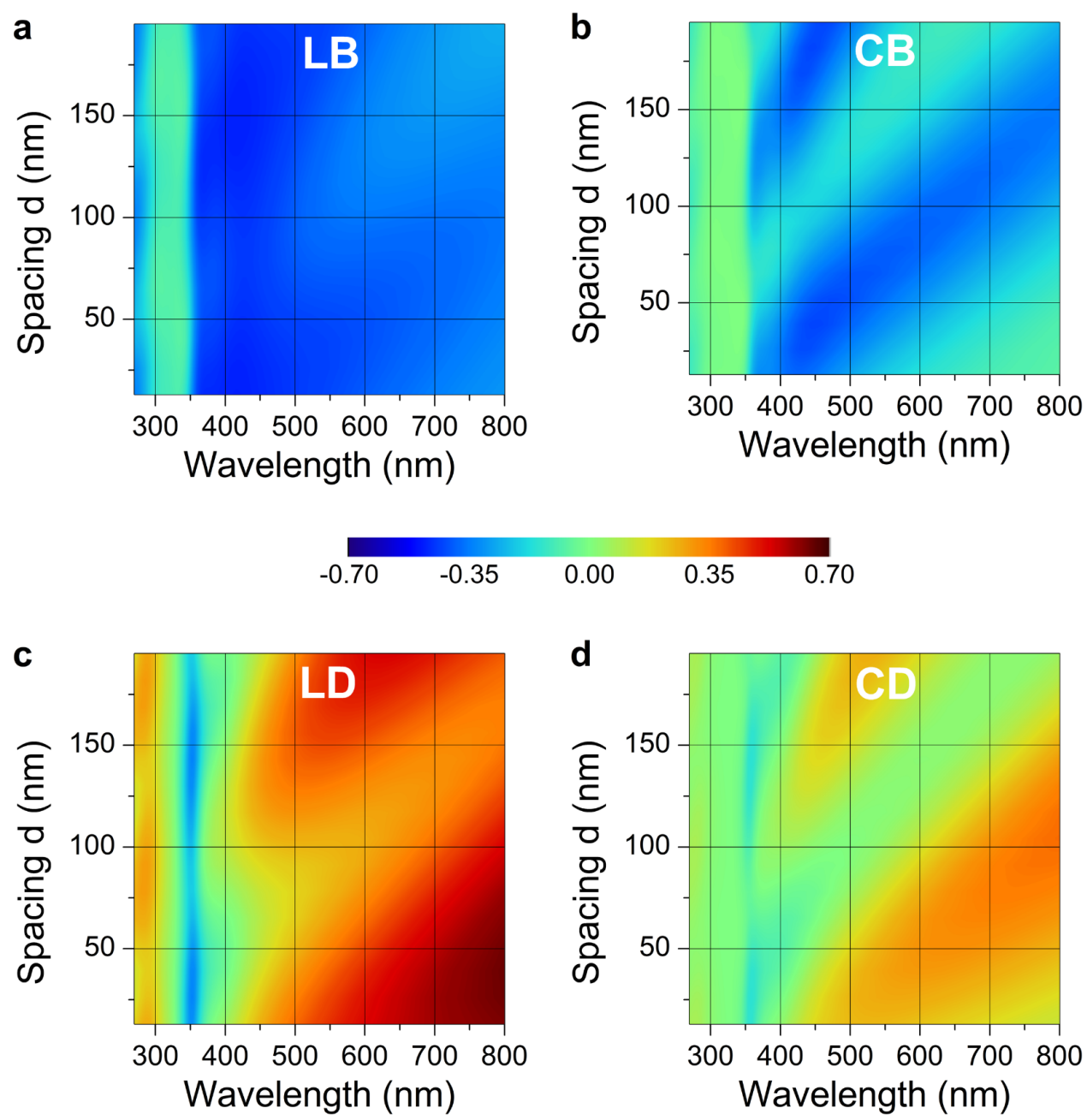

Figure S11. Color plot of the a) linear birefringence (LB), b) circular birefringence (CB), c) linear dichroism (LD) and d) circular dichroism (CD) spectra modeled using the transfer matrix approach for 2-layer samples oriented at a fixed angle $\left(\alpha=45^{\circ}\right)$ with a varying interlayer spacing $d$. The spectra are identical to those of Fig. $\mathbf{S 8}$ plotted with the same color scale as indicated. 
a

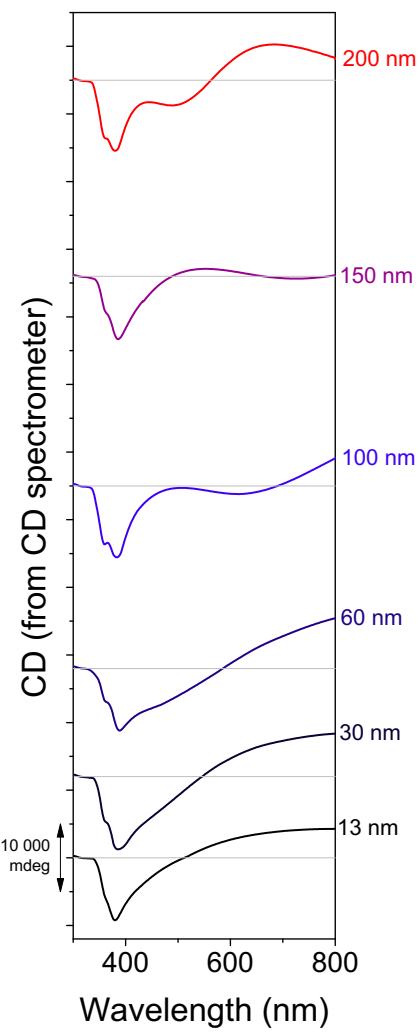

b

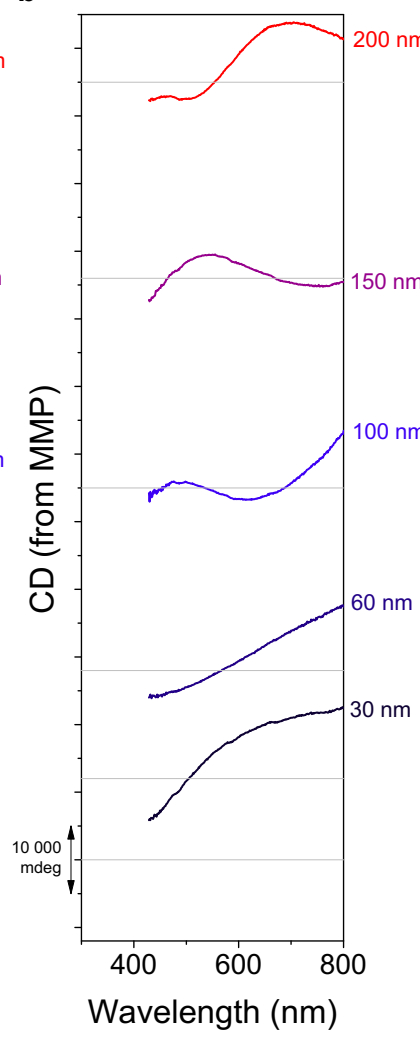

C

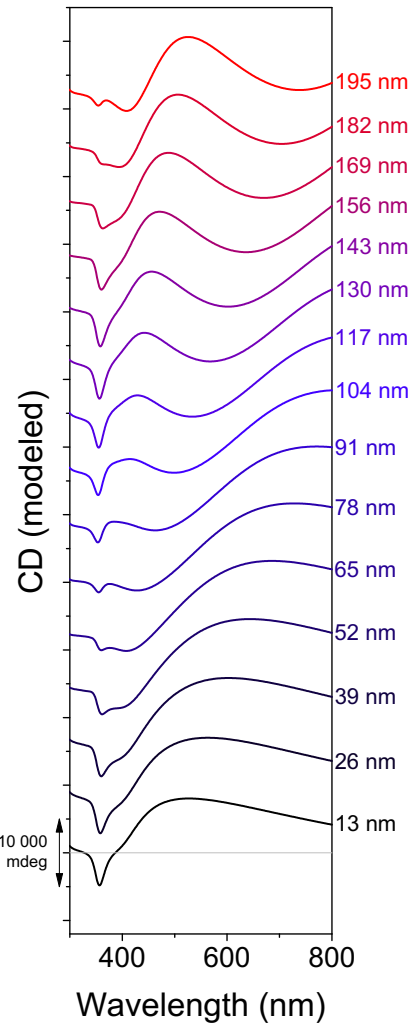

Figure S12. Circular dichroism (CD) spectra a) measured on a CD spectrometer, b) measured with a Mueller Matrix Polarimeter (MMP) and c) modeled using the transfer matrix approach for 2-layer samples oriented at a fixed angle $\left(\alpha=45^{\circ}\right)$ with a varying interlayer spacing $d$. The measured spectra by $C D$ spectroscopy are the same as those of Figure $3 c$ and the modeled $C D$ spectra are identical to those of Figure S8d and Figure S9d. The spacing between the measured CD spectra is proportional to the distance between the oriented AgNW layers and is consistent with the position of the modeled spectra.

\section{Supplementary references}

1. Arteaga, O.; Ossikovski, R., Complete Mueller matrix from a partial polarimetry experiment: the 12element case. J. Opt. Soc. Am. A 2019, 36, 416-427.

2. Schubert, M., Polarization-Dependent Optical Parameters of Arbitrarily Anisotropic Homogeneous Layered Systems. Phys. Rev. B 1996, 53 (8), 4265-4274.

3. Pérez, J. J. G.; Ossikovski, R., Polarized Light and the Mueller Matrix Approach. CRC Press, Taylor \& Francis Group: Boca Raton (USA), 2016; p 405.

4. Nichols, S.; Arteaga, O.; Martin, A.; Kahr, B., Measurement of transmission and reflection from a thick anisotropic crystal modeled by a sum of incoherent partial waves. J. Opt. Soc. Am. A 2015, 32, 20492057.

5. Nichols, S. M.; Arteaga, O.; Martin, A. T.; Kahr, B., Partially coherent light propagation in stratified media containing an optically thick anisotropic layer. Appl. Surf. Sci. 2017, 421, 571-577.

6. Arteaga, O.; Freudenthal, J.; Nichols, S.; Canillas, A.; Kahr, B., Transmission ellipsometry of anisotropic substrates and thin films at oblique incidence. Handling multiple reflections. Thin Solid Films 2014, 571, 701-705. 